\title{
Safety and Feasibility of Balloon-Assisted Embolization with Onyx of Brain Arteriovenous Malformations Revisited: Personal Experience with the Scepter XC Balloon Microcatheter
}

\author{
Bharathi D. Jagadeesan ${ }^{a-c}$ Andrew W. Grande ${ }^{a-c}$ \\ Ramachandra P. Tummala ${ }^{a-c}$ \\ ${ }^{a}$ Department of Radiology, University of Minnesota, Minneapolis, MN, USA; \\ ${ }^{b}$ Department of Neurosurgery, University of Minnesota, Minneapolis, MN, USA; \\ 'Department of Neurology, University of Minnesota, Minneapolis, MN, USA
}

\section{Keywords}

Balloon-assisted embolization · Onyx · Arteriovenous malformations · Embolization

\begin{abstract}
Background/Objective: Compliant dual-lumen balloon microcatheters have been used to perform balloon-assisted embolization (BAE) of brain arteriovenous malformations (AVMs) with ethylene vinyl alcohol copolymer (Onyx). However, vessel rupture and microcatheter retention have been reported from BAE using these microcatheters. Using an extra-compliant balloon microcatheter (Scepter XC; Microvention, Tustin, CA, USA) could help avoid pial vessel rupture during BAE. We herein report our experience using this balloon microcatheter for BAE. Methods: This retrospective study included patients who underwent BAE of brain AVMs at our institution between June 2012 and March 2017. Results: The extra-compliant Scepter XC balloon microcatheter was used for BAE of brain AVMs in 23 patients aged $44.3 \pm 16.7$ years (range 0-65 years). A total of 40 intracranial vessels (39 pial arteries and 1 pial vein) were catheterized and embolized during 30 separate sessions. In all instances, the balloon microcatheter could be successfully advanced to the AVM nidus. A mean volume of $2.4 \pm 1.7 \mathrm{~mL}$ (range $0.65-4.6 \mathrm{~mL}$ ) of Onyx was injected per session. There were no instances of vessel rupture, microcatheter retention, or stroke. Conclusion: Utilization of the extra-compliant balloon microcatheter results in safe and effective $B A E$, which adds to the growing experience with $B A E$ for AVM treatment.




\section{Introduction}

The use of dual-lumen balloon microcatheters for the delivery of ethylene vinyl alcohol copolymer (Onyx; Medtronic, Fridley, MN, USA) into brain arteriovenous malformations (AVMs) is a relatively recent development. In the majority of reported instances, the Scepter $\mathrm{C}$ balloon microcatheter (Microvention, Tustin, CA, USA) was used in this manner, and it has been speculated that balloon-assisted embolization (BAE) with Onyx could be associated with lower fluoroscopy times, good nidal penetration, and avoidance of potential complications from the reflux of Onyx [1-3]. However, there have also been reported instances of intracranial arterial rupture from inflation of the Scepter C balloon microcatheter in the distal intracranial circulation during embolization of brain AVMs [1,3]. Herein, we report our experience with using the more compliant Scepter XC balloon microcatheter for BAE in a series of patients with brain AVMs who underwent embolization over the past 5 years at our institution.

\section{Methods}

\section{Data Collection}

We retrospectively analyzed our prospectively maintained institutional database of endovascular surgical neuroradiology procedures to identify those brain AVM patients who underwent BAE with Onyx at our institution between June 2012 and March 2017. Institutional review board permission was obtained for this retrospective analysis. For each patient, basic demographic data were collected in addition to grade and presentation of brain AVM (symptomatic/asymptomatic; hemorrhagic/nonhemorrhagic), number of embolization procedures performed, method of procedural sedation, number and kind of balloon microcatheters used with each procedure, fluoroscopy times, total volume and type of Onyx injected, number and location of arterial feeders from which embolization was performed, complications with each procedure, and eventual management of brain AVM with surgical resection or radiosurgery.

\section{BAE Technique}

BAE procedures were carried out with the patient under conscious sedation or general anesthesia based upon operator preference. The $4 \times 11 \mathrm{~mm}$ Scepter XC balloon microcatheter was used in every instance where BAE was performed from a pial vessel. The tip of the microcatheter was steamed for approximately $10 \mathrm{~s}$ to avoid premature deflation; thereafter, the balloon microcatheter was purged of air using a $50 \%$ mixture of VISIPAQUE 320 and heparinized saline. This was achieved without ex vivo pre-inflation of the balloon by immersing the tip of the microcatheter in a container of heparinized saline, firmly holding the balloon segment between the operator's fingers during contrast mixture injection through the side port into the balloon lumen, and continuing the contrast mixture injection until no more air bubbles were seen to exit the tip of the balloon microcatheter. Once the air has been completely purged, further injection of contrast in vivo will always seal the purge hole as expected.

The balloon microcatheter was then navigated into the intracranial circulation in the usual manner, the appropriate arterial feeder for the brain AVM was selected, and the tip of the balloon microcatheter was advanced as close to the nidus as possible. In the majority of instances, an intermediate catheter was used to provide support for distal navigation of the balloon. Once the balloon microcatheter was determined to be in the ideal position, the balloon was gently inflated under digital roadmap guidance until its diameter matched that of the catheterized artery. Microcatheter angiography was performed before and after balloon inflation to confirm appropriate positioning of the microcatheter tip and to study the changes in flow dynamics with balloon inflation (in instances where an en passage artery became visible with microcatheter injection before or following balloon inflation, embolization was not carried out from that particular location). The location of the distal and proximal markers of the balloon was marked on the DSA display screen. BAE was then commenced with Onyx in the previously described manner. Embolization was interrupted if there was reflux of Onyx more than halfway along the balloon and abandoned if there was persistent Onyx reflux beyond the halfway mark on resumption of injection. Once satisfactory embolization had been achieved, the balloon was deflated and the Scepter XC microcatheter was removed with gentle negative suction at the hub of the microcatheter injection lumen. 

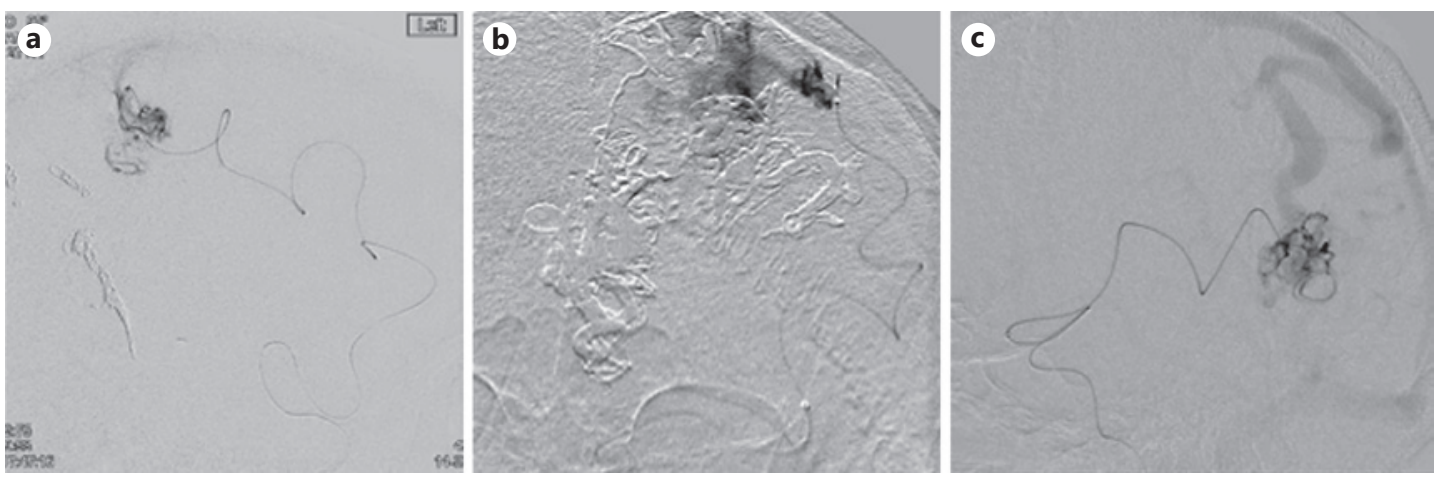

Fig. 1. Distal navigability of the Scepter XC balloon microcatheter into arterial feeders for brain arteriovenous malformations situated at the A4 (a), M4 (b), and P4 (c) segments of the intracranial circulation in three different patients. c Of note, the microcatheter was navigated from the internal carotid artery via the posterior communicating artery into the posterior circulation.

\section{Results}

BAE with the Scepter XC balloon microcatheter was performed in 23 patients (12 male and 11 female) aged $44.3 \pm 16.7$ years (range $0-65$ years). The majority of patients $(15 / 23)$ had Spetzler-Martin grade III AVMs, and 10 presented with hemorrhage; 2 children had vein of Galen aneurysmal malformation. A total of 30 embolization procedures were performed on these patients, and a total of 40 intracranial vessels ( 39 pial arteries and 1 pial vein) were catheterized for injection of Onyx through the balloon microcatheter in these sessions. In every instance, the balloon microcatheter could be successfully advanced to the nidus of the AVM (Fig. 1). A mean volume of $2.4 \pm 1.7 \mathrm{~mL}$ (range $0.65-4.6 \mathrm{~mL}$ ) of Onyx was injected in each instance (Onyx 18 was injected in all instances, except for 2 sessions during which Onyx 34 was used). The mean fluoroscopy time per $\mathrm{mL}$ of Onyx injection was $23.3 \mathrm{~min}$. There was no incidence of vessel rupture, catheter retention, or clinically relevant ischemic or hemorrhagic stroke (no instances of contrast extravasation or occlusion of nontarget vessels on angiography either). The nidal penetration with Onyx was subjectively felt to be satisfactory in every instance. Except for the children with vein of Galen malformation (Fig. 2), all other patients underwent either surgical resection or stereotactic radiosurgery after their embolization procedures.

\section{Discussion}

BAE for the treatment of brain AVMs is still evolving and its position in the armamentarium of the ESN practitioner is not yet well established. When compared to traditional embolization techniques, it offers potential advantages by preventing complications associated with the reflux of Onyx along the microcatheter tip, such as microcatheter retention, stroke from embolization of en passage arteries, hemorrhage from difficult microcatheter extraction, etc. [1-4]. In addition, it has been reported that BAE could result in lower fluoroscopy times and better nidal penetration when compared to traditional embolization techniques [5]. In theory, some of these advantages should persist even when BAE is compared to the use of detachable tip microcatheters, since in the latter case reflux of Onyx can still occur and result in strokes etc., although the microcatheter tip can be safely detached. 

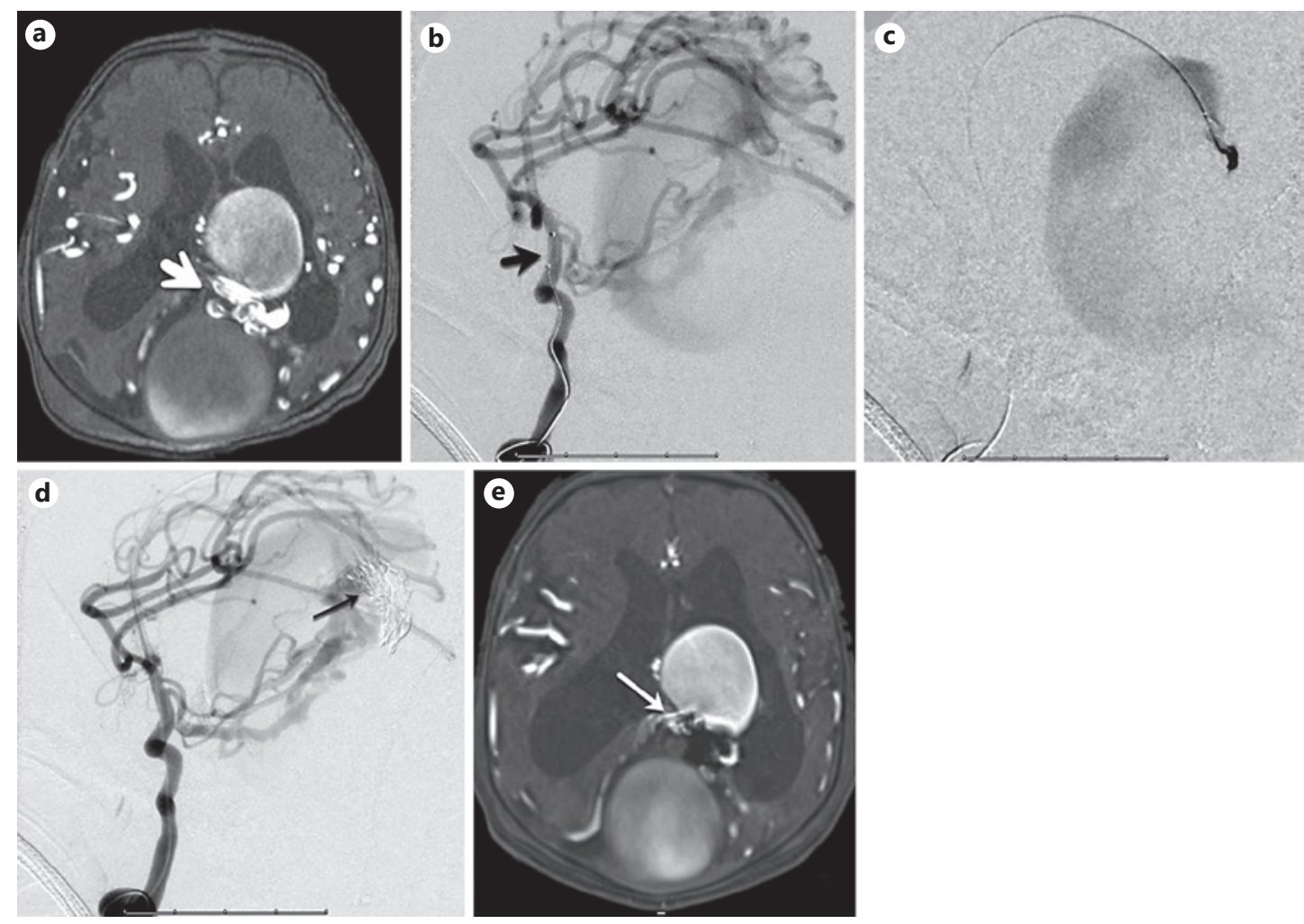

Fig. 2. a Axial image from a time of flight magnetic resonance angiography in a 1-day-old child with a choroidal vein of Galen aneurysmal malformation showing enlarged posterior callosal arteries (arrow) supplying the malformation (the large venous pouch can also be seen). b The Scepter XC (arrow) being navigated from an umbilical artery approach into the left internal carotid artery at 3 days of age, as seen on this lateral projection from a DSA. c Microcatheter injection through the Scepter XC, which has been navigated distally into the posterior pericallosal arterial segment with opacification of the venous pouch. $\mathbf{d}$ Onyx cast (arrow) from injection through the Scepter XC (which can still be seen). Note the penetration of the nidus without penetration into the venous pouch. e Axial time of flight from postoperative magnetic resonance angiography shows Onyx cast (arrow) from the procedure.

To date, these potential advantages with BAE have not been reported in a large number of patients. On the contrary, the existing reports on BAE document instances of balloon microcatheter retention and arterial rupture from balloon inflation, both of which could have disastrous consequences for the patient $[1,2]$. Notably, the majority of patients included in these existing reports underwent $\mathrm{BAE}$ with the Scepter $\mathrm{C}$ balloon microcatheter or in rare instances the Ascent balloon microcatheter [6]. At our institution, we perform BAE exclusively with the Scepter XC balloon microcatheter for all pial embolizations and we have not experienced these complications.

We have also modified the ex vivo and in vivo steps involved in preparation of the microcatheter and the technique for BAE. Specifically, by avoiding ex vivo pre-inflation of the balloon, we have been able to successfully track the Scepter XC balloon microcatheter in distal and tortuous intracranial vessels, and by steaming the tip of the microcatheter prior to its introduction into the bloodstream, we have been able to avoid premature deflation of the balloon. Of note, although the manufacturer's instructions for use recommend steaming for $20 \mathrm{~s}$, in our experience, steaming the tip for $10 \mathrm{~s}$ in enough to avoid premature deflation. It is important to use this technique to avoid premature deflation since this 
phenomenon, if undetected, can catch the operator unawares and result in reflux of Onyx and entrapment of the microcatheter or embolization of en passage arteries. Once the balloon microcatheter is in position and ready for BAE, we also mark the location of the balloon markers on our DSA display monitors to help us understand the extent of Onyx reflux if there is any. We do so, since in our experience, reflux of Onyx can still occur with the balloon fully inflated if resistance to antegrade flow of Onyx is very high. Therefore, we do not assume that inflation of the balloon offers us absolute protection against reflux, and we do not tolerate Onyx reflux proximal to the halfway point of the interval between the balloon markers.

By strictly adhering to these technical modifications when using the Scepter XC balloon microcatheter for BAE, we have entirely avoided arterial rupture and balloon microcatheter retention in our patients. The safety of balloon inflation with the Scepter XC balloon in these small vessels is perhaps best illustrated by the successful performance of retrograde transvenous embolization from a pial vein in a 1-year-old with a vein of Galen aneurysmal malformation as reported elsewhere [7]. In our experience, it is safe to carefully inflate the Scepter $\mathrm{XC}$ balloon in vessels $\geq 1.5 \mathrm{~mm}$ in diameter, provided this is performed slowly under digital roadmap guidance until the balloon diameter barely matches the vessel diameter. We have also encountered no difficulties in tracking the balloon microcatheter to the desired location for BAE. Admittedly, our ability to successfully track the Scepter XC to the desired location is influenced by an a priori assessment of the feasibility of such maneuvers based on the vascular anatomy. However, as can be evidenced from the accompanying images (Fig. 1), we did successfully track these microcatheters quite distally into the intracranial circulation.

There are some drawbacks and limitations to our report. Most importantly, it is retrospective in nature, and we did not perform a direct comparison between BAE and other methods for Onyx injection in a randomized and prospective manner. Unless such a study is performed on a much larger number of patients, rigorous scientific proof for the advantages of BAE is not possible. We also did not directly compare metrics such as fluoroscopy times and volumes of Onyx injected between patients undergoing BAE and those having more traditional embolization. In our opinion, such a comparison is difficult, given wide variations in AVM angioarchitecture, arterial tortuosity, embolization goals, and nidal anatomy between individual patients. We have also not reported the extent of obliteration of AVM nidus with each embolization session with the BAE technique. In part, this is a deliberate omission since such quantification is often subjective (not based on true volumetry of total volume of the AVM nidus and volume of the embolized part of the AVM nidus). Also, we have taken into consideration that the goal of AVM embolization is often not purely volume reduction, but rather more strategic and aimed at embolization of parts of the nidus that harbor microaneurysms thought to be responsible for hemorrhage or embolization of those parts of the nidus that are thought to be difficult to resect surgically. Additionally, we do not report here the selection criteria used to select patients for preoperative embolization, since that is outside the scope of this paper.

In conclusion, BAE can potentially be safely performed in brain AVMs using the Scepter $\mathrm{XC}$ balloon microcatheter.

\section{Statement of Ethics}

This research was conducted with approval from the Institutional Review Board of the University of Minnesota (STUDY00000593). 


\section{Disclosure Statement}

Dr. Jagadeesan is a consultant for Microvention, the manufacturers of the Scepter XC balloon microcatheter mentioned in this paper. He did not receive any financial assistance for the preparation of this paper. Dr. Grande and Dr. Tummala have no competing interests. This research received no specific grant from any funding agency in the public, commercial, or not-for-profit sectors.

\section{Author Contributions}

B.D. Jagadeesan: concept, study design, data analysis, manuscript preparation. A.W. Grande: critical review and manuscript revision. R.P. Tummala: critical review and manuscript revision.

\section{References}

1 Jagadeesan BD, Grigoryan M, Hassan AE, Grande AW, Tummala RP: Endovascular balloon-assisted embolization of intracranial and cervical arteriovenous malformations using dual-lumen coaxial balloon microcatheters and Onyx: initial experience. Neurosurgery 2013;73(2 Suppl Operative):ons238-ons243; discussion ons243.

2 Spiotta AM, James RF, Lowe SR, Vargas J, Turk AS, Chaudry MI, et al: Balloon-augmented Onyx embolization of cerebral arteriovenous malformations using a dual-lumen balloon: a multicenter experience. J Neurointerv Surg 2015;7:721-727.

3 Paramasivam S, Niimi Y, Fifi J, Berenstein A: Onyx embolization using dual-lumen balloon catheter: initial experience and technical note. J Neuroradiol 2013;40:294-302.

4 Orozco LD, Luzardo GD, Buciuc RF: Transarterial balloon assisted Onyx embolization of pericallosal arteriovenous malformations. J Neurointerv Surg 2013;5:e18.

5 Gentric JC, Raymond J, Batista A, Salazkin I, Gevry G, Darsaut TE: Dual-lumen balloon catheters may improve liquid embolization of vascular malformations: an experimental study in swine. AJNR Am J Neuroradiol 2015; 36:977-981.

6 Fifi J, Niimi Y, Berenstein A: Onyx embolization of an extensive mandibular arteriovenous malformation via a dual lumen balloon catheter: a technical case report. J Neurointerv Surg 2013;5:e5.

7 Jagadeesan BD, Zacharatos H, Nascene DR, Grande AW, Guillaume DJ, Tummala RP: Endovascular management of a vein of Galen aneurysmal malformation in an infant with challenging femoral arterial access. J Neurosurg Pediatr 2016;18:231-234. 